\title{
Superstrings Theory
}

\section{Solomon Budnik \\ budnik1@013.net}

Abstract: This article portrays space and time as conformal projections of motion, and reveals the co-relation between the universality of space-time (relativistic model) and energy-frequency (quantum model). This new theory of astrophysics proves that unified matter of coupled superstrings created a supercavity previously thought to be a black hole, thus negating the assumption of a gravitational collapse of a star to form a black hole. In general, there is no cosmic hole in the fabric of space where the macroscale density of space and not the microscale density of matter should be considered. What was previously conceived as a black hole is a self-gravitating torus (see ill. below) - a cosmic supercollider of doubly elliptic strings manifold, and this explains the origin of relativistic jets from magnetized accretion disks, as elaborated below.

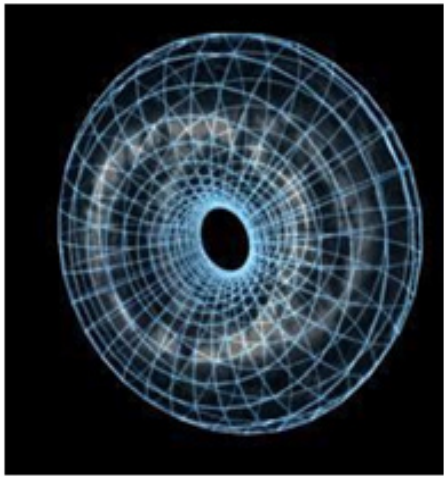

Clifford torus

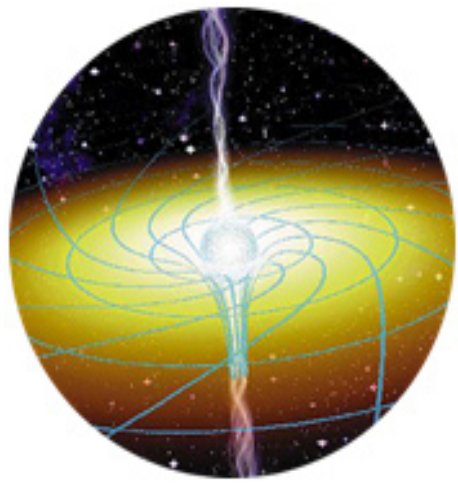

Hydrodynamic simulation of rotating black hole

(by quantum gravity lab, Nottingham univ.)

Accordingly, this chapter contributes to the understanding of the formation of twin self-gravitating accretion discs by twin superstrings in gauge/gravity duality, where at $D=26$ the string dynamics is that of harmonic oscillators, as was predicted earlier by duality theorists. Hence, our new cosmic model is in conformity with the basic principle of duality in nature, where no singular event of the alleged Big Bang could had taken place, for there must always be the cause and the effect in a dual interaction, as in the wave-particle duality.

\section{THEORY OF QUANTUM GRAVITY IN SUPERSTRINGS}

Two parallel lines never meet in geometry, but superstrings of matter in cosmos might couple and converge in a bow-like superstructure with a shock wave, as in fig. 4 on p. 36 and ref. 2 on p.39. Compare, on earth a pair of lines of longitude is parallel at the equator but converge toward the poles. So, let's consider two parallel superstrings of matter with integrated accretion discs in the middle of each one. (Fig. 1 on p. 33) Those discs are formed by two coupled linear superstrings of matter, whose shared central core inflates to create an accretion disc (fig. 1) in a quasi super-Planckian effect (fig 2.) 


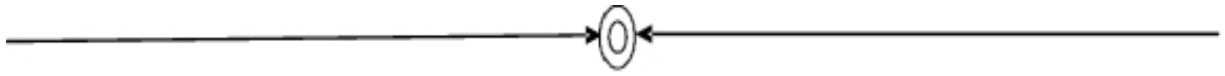

Fig1. Coupled Superstrings

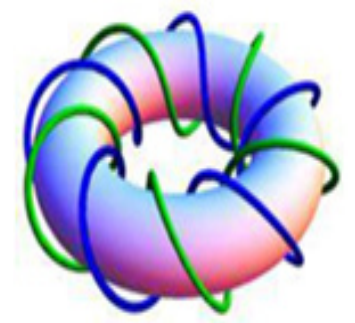

Fig2. Accretion disc of coupled superstrings

The accretion disc of matter in fig. 1 and 2 consists of nuclei (see ref. 3 on p. 40) and membrane that oscillate in unison in unified resonant frequency in a given frame of space-time, which is not trapped in geometrical dimensions, but oscillates/modulates in different atomic frequencies we cannot perceive or detect, for we're not yet tuned to them, as a common radio cannot pick up radio waves it is not tuned to. Therefore, our superstrings model and concept differ from the string theory model, which envisages a structure of different integrated, not unified and inharmonic shapes, which cannot co-vibrate in common pitch. (For pitch see ref. 3)

In our opinion, a cosmic superstring resembles a hexagonal musical string which has a "core» of one material, and is encircled by other material. A musical string is linearly stretched along the fingerboard of a musical instrument, where the fingers of the musician press/short them in different intervals, while the bow pulls the string sideways to make it vibrate in various sounds. Similarly in cosmos, a superstring of matter stretches itself linearly in space where applied gravity presses/shorts it in spacetime intervals/knots of matter and then pulls it sideways to make it resonate/oscillate in various frequencies and gravitational waves that create a supercavitation (not a black hole) in space caused by the density of coupled superstrings, as in fig. 1 .

Accordingly, our superstring is not a macrojet of matter but a chain of intertwined gauge/gravity microstrings -- cosmic tubes that funnel the invisible superfluid dark matter (as continuum fluid in the phase space) and highly compressed energy in thereby twisted spacetime (Fig. 3 a, b), which expands and contracts in pulsation due to inherent gravity and fluorescent mirror reflections of subatomic particles.

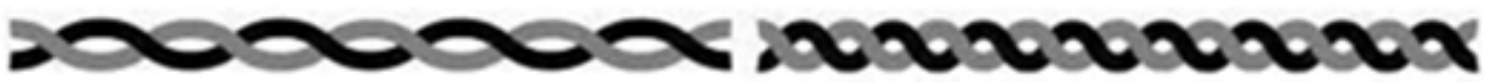

(a)

Fig. 3, (a, b)

These are chains of microstrings in twisted timespace as time and space woven together per Einstein's theory of relativity. Our concept image of intertwined/woven superstring fabric in fig. 3 has its further confirmation in ref. 4 on p. 41.

So in our unified model, coupled superstrings unify in space via supercore (fig. 1 above) of the overlapping, oscillating condensed matter (fig. 2 above), which creates in supercavitation a superdensity vortex, previously termed the black hole that had puzzled scientists who couldn't comprehend how matter spiralling around a black hole in an accretion disk suddenly plunges into the void without gravitational collapse.

Nota bene: the intertwined superstrings in fig. 3 above have open opposite ends through which matter traverses spacetime in superconductivity contrary to the speed of light constant, for said superstrings acts as a supercollider and superaccelerator in space. The inherent shape of our superstrings with integrated succession 
of multiple micro-barriers in supercold vacuum chambers explain the phenomena of neutrinos that propagate unobstructed through space and earth, originate in one part of the universe and immediately appear in a remote part by piercing spacetime. For neutrinos the spin is always opposite the linear momentum, so in our superstring accelerator we might deal with the Casimir effect and particles that emit the virtual photons which lose momentum $\mathbf{p}$ in the recoil, and the other particles get the momentum.

Vacuum neutrino oscillations are possible in relativistic wave packets per quantum field theory. Neutrino is considered as wave packets either in QM or in QFT. QM postulates the wave function of neutrino and this is the source of numerous paradoxes. Standard S-matrix theory of QFT works with states of definite momentum -neutrinos uniformly distributed over all space in the propagation momentum in phase optimization per Wave Field Theory.

The neutrino state is a superposition of massive neutrino wave packets determined by the production process where the energies and momenta of the massive neutrino components relevant for neutrino oscillations are in general different from the average energies and momenta of the propagating massive neutrino wave packets, as in ourmodel of the cosmic neutrinos accelerator.

(See C. Giunti, Neutrino Wave Packets in Quantum Field Theory, 2002. D. V. Naumov and V. A. Naumov, Vacuum neutrino oscillations with relativistic wave packets in quantum field theory. J. Phys. G 37 (2010) 105014, Dmitry V. Naumov, 2011).

In re: Physicists may have linked the elusive source for the highest energy neutrinos, abundant sub-atomic particles with no electrical charge that race through the universe to a supermassive cluster of matter at the center of the Milky Way galaxy called Sagittarius A\|.

Observations leading to a potential breakthrough in the identification of a source were made with three NASA space telescopes, the 15-year-old Chandra X-ray Observatory, 10-year-old Swift Gamma Ray Burst mission observatory and the 2-year-old NuSTAR (an X-ray observatory), as well as the IceCube Neutrino Observatory, which is positioned under the South Pole. IceCube has recorded 36 high-energy neutrinos since the facility becameoperational in 2010. "We now have the first evidence that an astronomical source, the Milky Way's supermassive black hole may be producing these very energetic neutrinos," states University of Wisconsin physicist Yang Bai, in a Nov. 13 NASA announcement. He is among a half dozen scientists from the universities of Wisconsin and Hawaii who published their findings in Physical Review D.

The idea is that albeit a neutrino has no single mass, by passing through each termonuclear combustion chamber in our superstring, a swarm/block of neutrinos accelerates in jets (see ref. 3) with each fusion cycle and accumulates enormous cumulative energy to warp the spacetime where the entry point is the instant exit point for neutrinos in any part of the universe. Hence, the protouniverse might have been built by such blocks of primordial matter to then evolve into our universe per my theory of dual (non-parallel) universes.

We can accordingly claim that condensed water is the end product of fusion in cosmic accelerator described above, i.e., when the positron passes through a cloud chamber it simply doesn't have enough time around any particular electron. What it does do, however, is to ionize some of the atoms which, in turn, allows the super saturated water vapour to condense along the path in our twin superstrings -- twin black holes integrated system as in fig. 4 on p. 36.

With regard to the unified matter model in fig. 4 below, local quantum entropy there had reached its saturation point beyond Von Neumann entropy and Lev Landau* state vector limits, so the ends of the upper linear string of matter in our model were curved and gravitationally pulled/shifted (for shift see ref. 3) downward to the corresponding ends of the linear base string of matter, creating thereby a bow of unified matter (fig. 4 and ref. 2 below) with the jet arrow of matter emitted along the axis of rotation from the base disc via upper disc over its event horizon, as shown in fig. 4. 
So in our bow of matter model we deal with the induced curvature of space per theory of general relativity, which predicted that a sufficiently compact mass can deform the space-time. *Landau, L. (1927). "Das Daempfungs problem in der Wellenmechanik". Zeitschrift für Physik. 45 (5-6): 430-464

Large vertical arrow in our model represents a relativistic jet of matter which erupts from the twin superstrings structure due to accumulated tension applied by the bow of unified matter and rotational acceleration around the axis of the disc core. The 2009 experiments conducted by Professor Sergey Lebedev's team in the Department of Physics at Imperial College London (www.imperial.ac.uk) confirm our theory and model by suggesting that the jets of matter are fired out more like bullets or buckshot: they don't break into pieces -- they are formed "in pieces."

And that in our opinion could be the anti-gravitational fall up of anti-matter.

Note that our jet-arrow of matter points in one upward direction and isn't emitted in the opposite direction as suggested by the astronomers in ref. 3 on p. 40. In fact, the info in ref. 3 confirms our twin accretion disks concept as shown in fig. 4 below, where the jet of matter from invisible lower disc of dark matter is emitted via upper visible twin disc, in accordance with the constant of duality in the nature and cosmos. (Compare with twin galaxies or single ones after separation).

The shown in fig 4 bow of matter model has its reflection in a bow-like shock wave in cosmos in NASA image (see photo in ref. 3 on p. 40).

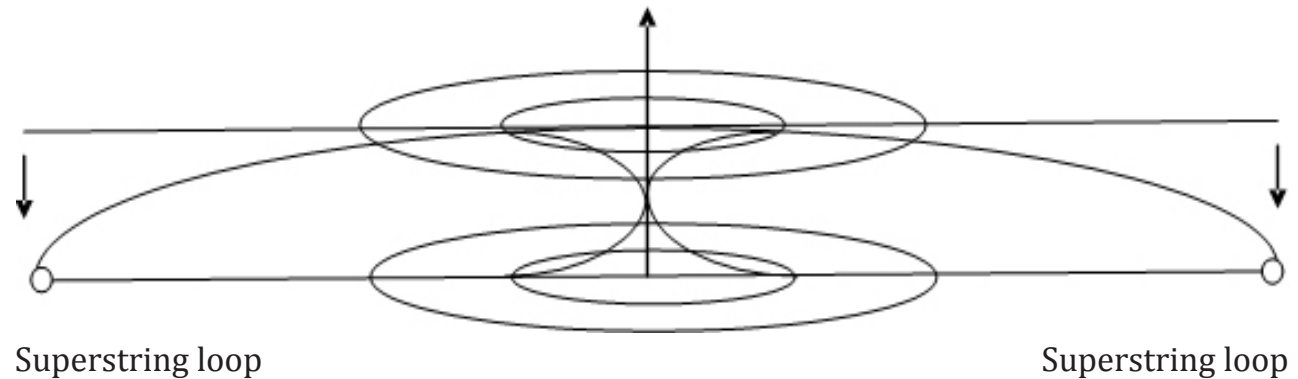

Fig4. Bow of Matter Model

Twin parallel superstrings with twin accretion discs in our bow of matter model above are created by doubly elliptic strings (fig. 5 below) and function as toroidal "saddle" space (fig. 6) and dynamic horn tori with a uniform grid (fig. 7 on p. 38), where matter from the upper accretion disk in our model is forced downward to the lower accretion disk by Coriolis force, to then erupt upward in a jet of matter via upper disk due to accumulated density, energy and pressure.

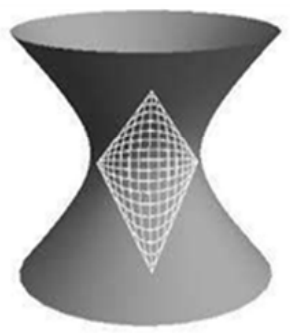

Fig5. Doubly Elliptic Strings

Our model of matter above fits the geometry of the universe which is "open" or negatively curved like a saddle, according to a new model proposed by researchers in Europe who have studied anomalies in the cosmic microwave background radiation. The anomalies were first detected by NASA's Wilkinson Microwave Anisotropy Probe (WMAP) in 2004 and were confirmed earlier this year by the European Space Agency's Planck space mission. 


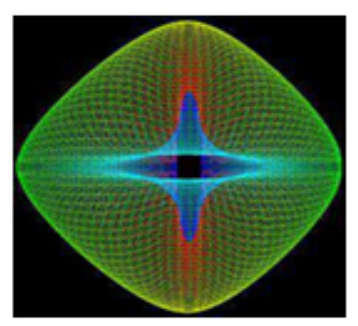

Fig6. Toroidal saddle space, integrating self-gravitating torus previously mistaken for a black hole.

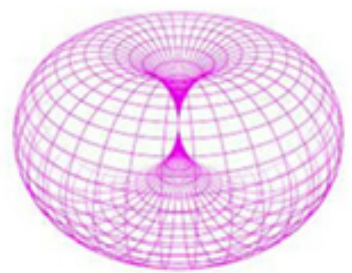

Fig7. Dynamic horn torus

We further elaborate that toroidal shape of matter has no central "hole" and is not a "black hole" per se, since it folds in upon itself (general relativity) and all points along its surface converge into a zero-dimensional point at the center called the vertex, which acts as a particles trap where particles collide and interact.

One might ask how the gravitational pull and the bow o matter was created in the parallel superstrings of matter in our model (fig. 4, p. 40 above), with no external force applied. The answer is that we deal here with the warps of matter as the set of lengthwise streams of particles (fig.3, p.38 above) held in opposing tension in said strings and twisting around those strings of matter. Quantum electrodynamics in quantum superposition is applied here, for two parallel strings of matter attract or repel themselves depending on whether the energy flows in the same or opposite directions in mutual action, where two lengths of matter-carrying strings is proportional to their lengths and to the intensities of their currents, to paraphrase the Ampere's law.

Our warp concept is supported by a practical experiment conducted by Professor Sergey Lebedev's team in the Department of Physics at Imperial College London that sent a high-powered pulse of energy into an aluminium disk. In less than a few billions of a second, the aluminium began to evaporate, creating a cloud of plasma very similar to the plasma cloud surrounding a young star. Where the energy flowed into the center of the disk, the aluminium eroded completely, creating a hole through which a magnetic field from beneath the disk could penetrate. The field initially pushes aside the plasma, forming a bubble within it. As the field penetrates further and the bubble grows, however, the magnetic fields begin to warp and twist, creating a knot in the jet.

\section{CONCLUSION}

To discuss further our superstrings of matter, we come to the notion of repellent gravity, as observed in galaxies. Due to anti-gravity between two superstrings of matter in our model, matter shifts toward the density points/ loops at the ends of unified strings. (For shifting see ref. 3 below). This corresponds with Max Planck's idea of a harmonic oscillator (classically, anything that wiggles like a mass bobbing on the end of an ideal spring). Hence, our superstrings are plugged at their low density vertex, representing a plasma conductor in a vacuum loop that can withstand large gravitational pull. Dynamics of plasma are often the sources of electromagnetic fields. ((Loop quantum gravity and Aharonov-Bohm effect might be applicable). Our superstrings model corresponds also with the superfluid vacuum theory (SVT).

Moreover, our bow-like superstrings model in fig 4, p.36 provides the "quantum bridge» between contracting and expanding matter where the fragmentation of the horizon by superimposed superstrings creates loops that accrete and bind in collapsing matter perturbations, since the string tension is less than a critical value and the loops are large-scale. 


\section{REF. 1}

\section{Twin Black Holes Discovered}

DPosted October 3rd, 2012

Astronomers recently made a surprising find while searching for a unique black hole in a tight cluster of stars 10,000 light years away from Earth. Instead of finding one black hole, scientists with the National Radio Astronomy Observatory (NRAO) found two twins, something that surprised them because, according to modern theory, there should only be one black hole in a cluster.

REF. 2

\section{Supernova-powered bow shock creates cosmic spectacle}

12:31 21 January 2013

Picture (below) of the DaySpace. Victoria Jaggard, space and physical sciences news editor

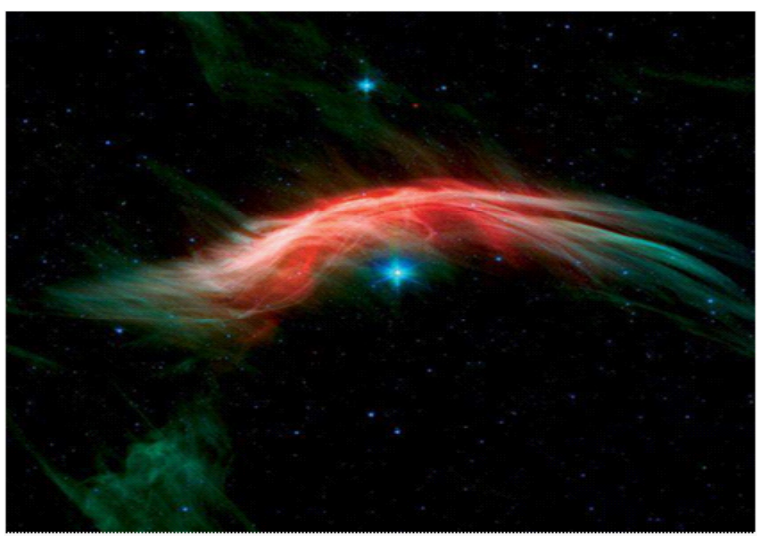

(Image: NASA/JPL-Caltech)

(Image: NASA/JPL-Caltech)

REF. 3

2013: Using the European Space Agency's

(ESA) XMM-Newton observatory, lead author Dr. María Díaz Trigo and colleagues studied a black hole binary system located in our own galaxy. This system, called 4U1630-47, has been known to show X-ray outbursts over the period of several months or even years, the agency explained in a statement. "In our observations, we found signs of highly ionized nuclei of two heavy elements, iron and nickel, said Trigo, who works at the European Southern Observatory in Munich, Germany. The discovery came as a surprise - and a good one, since it shows beyond doubt that the composition of black hole jets is much richer than just electrons."

"Intriguingly, we found the lines were not where they should be, but rather were shifted significantly said Dr. James Miller-Jones, who led the radio observations and is a member of the International Centre for Radio Astronomy Research (ICRAR). That shift was similar to that of the pitch change of a vehicle's siren as it moves towards or away from the hearer, and signified that the length of the sound wave is becoming shorter or longer due to the movement. The phenomenon led the study authors to believe that the particles were being accelerated to high speeds while in the jets - one towards the Earth, and the other in the opposite direction. According to Dr. Miller-Jones, this is the first strong evidence of the presence of such particles in typical black hole jets. -We've known for a long time that jets contain electrons, but haven't got an overall negative charge, so there must be something positively charged in them too, he explained." 
"Until now it wasn't clear whether the positive charge came from positrons, the antimatter "opposite" of electrons, or positively charged atoms, the Dr. Miller-Jones added. -Since our results found nickel and iron in these jets, we now know ordinary matter must be providing the positive charge."

Read more at http://www.redorbit.com/news/space/1113003040/black-hole-jet-contents-111413/\#i7XX4s DVdvHdPqrQ.99

REF. 4

Monster black hole from early cosmos challenges physics.

Thursday, 26 February 2015 Genelle Weule and Stuart Gary, ABC, see picture below.

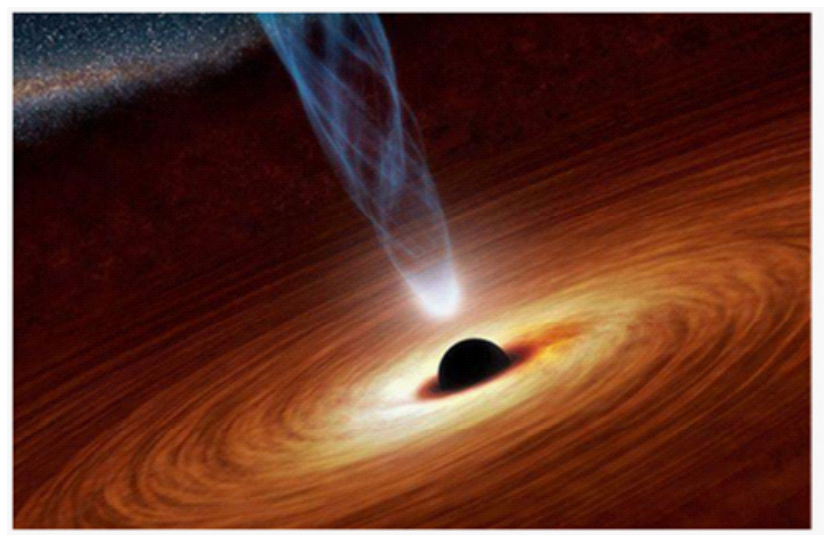

"New physics are needed to explain an ancient supermassive black hole quasar 12 billion times the mass of the Sun (NASA/Caltech). The discovery of a supermassive black hole from the early cosmos is set to rewrite physics, say scientists".

An international team of astronomers detected a black hole 12 billion times the mass of our Sun, they reported in the journal Nature. The black hole is the source of a powerful beam of bright material known as a quasar.

"When we found this supermassive black hole we got very excited because we had found something that we never thought we could find," says Dr Fuyan Bian of the Australian National University.

The team, led by Xue-Bing Wu at Peking University, discovered the black hole and quasar -- known as SDSS J0100+2802 -- using the Sloan Digital Sky Survey, then followed up with three other telescopes. With a luminosity of 420 trillion that of our Sun's, the new quasar is seven times brighter than the most distant quasar known. "This quasar is very unique. Just like the brightest lighthouse in the distant universe, its glowing light will help us probe more about the early Universe," says Wu.

Citation: Solomon Budnik, "Superstrings Theory". American Research Journal of Electronics and Communication Engineering; vol. 1, no. 1; pp: 32-38.

Copyright (c) Solomon Budnik. This is an open access article distributed under the Creative Commons Attribution License, which permits unrestricted use, distribution, and reproduction in any medium, provided the original work is properly cited. 